4. Ten Cate O. Nuts and bolts of entrustable professional activities. J Grad Med Educ. 2013:5(1):157-158.

5. Ten Cate O, Scheele F. Competency-based postgraduate training: can we bridge the gap between theory and clinical practice? Acad Med. 2007;82(6):542-547.

6. Shaughnessy AF, Sparks J, Cohen-Osher M, Goodell KH, Sawin GL, Gravel J Jr. Entrustable professional activities in family medicine. J Grad Med Educ. 2013;5(1):112-118.

\section{PRIMARY CARE RESEARCH PRIORITIES IDENTIFIED AND SHARED WITH STAKEHOLDERS}

This past spring, NAPCRG shared its views on what direction funding agencies should take when determining priorities in primary care research.

In the United States, letters were sent to leadership at the Agency for Healthcare Quality and Research (AHRQ) and the Patient-Centered Outcomes Research Institute (PCORI), and in Canada letters were sent to the Canadian Institutes of Health Research and Fonds de recherché du Quebec - Sante.

The following summarizes the priorities outlined for the United States as included in the letter sent to AHRQ and PCORI.

\section{Primary Care Research Through Practice-based Research Networks (PBRNs)}

The majority of research funding supports research of 1 specific disease, organ system, cellular or chemical process, and is not related to issues surrounding the total needs of a real life patient in primary care. Not only does the majority of health care take place in the primary care setting, this setting is the key interface between the patient and the primary care provider. The importance of what happens in that space is crucial to improving care, improving outcomes, reducing errors, and realizing meaningful patient-centered outcomes research. NAPCRG sees an unmet need in strong funding support for research that is conducted with and by primary care practices and their patients-essentially what PBRNs do.

\section{Practice Transformation}

Very little is known about important topics such as how primary care services are best organized, how new technologies impact care, how to maximize and priori- tize care, how to introduce and disseminate new discoveries so they work in real life, and how patients can best decide how and when to seek care. We know from our members and our patients that the need is great to understand what works for patients and practices. Part of this transformation includes the establishment of and reliance on interprofessional teams for training and patient care. More research into best practices related to this integration is needed in both the training and practice arenas. Transforming primary care practices to be effective medical homes for our patients should be a key priority - and one that can only be accomplished with studies in the primary care environment.

\section{Patient Quality and Safety in Non-Hospital Settings}

We are all aware of the research related to the many improvements in patient care in hospital settings, and the continued work in this area. Our patients tell us that one of the key areas that is problematic for them is in the non-hospital setting. For example, the communication between specialist and patient and primary care provider is an area that needs work to understand how to improve. Improved methods for engaging patients in the management of their health conditions is a key area that needs further study. Patient-centered outcomes research informs the evidence needed for guidelines that we can trust, but the voice of patients in the development of clinical practice guidelines remains a promise unfulfilled.

\section{Multimorbidity Research}

More attention and research needs to be directed to the "real-life" patient; the one who doesn't have diabetes alone, for example, but also has cardiovascular disease, as well as renal disease. In 2000, an estimated 60 million Americans had multiple chronic conditions. By 2020, an estimated 81 million people will have multiple chronic conditions. In addition, care for people with chronic conditions is expected to consume $80 \%$ of the resources of publicly funded health insurance programs by 2020 . When private and public expenditures are combined, $51 \%$ of total expenditures are for those with multiple chronic conditions. ${ }^{1}$ More research funding and attention needs to be directed at multimorbidity research.

\section{Mental and Behavioral Health Provision in Communities and Primary Care Practices}

Research addressing best practices for integrated mental and behavioral health provision in communities and primary care practices, and ways to increase the uptake of these models in primary care practices is needed. As a 2011 Robert Wood Johnson policy brief states, 
"Comorbidity between mental and medical conditions is the rule rather than the exception. In the 2003 National Comorbidity Survey Replication (NCS-R), more than $68 \%$ of adults with a mental disorder had at least 1 medical condition, and $29 \%$ of those with a medical disorder had a comorbid mental health condition. Moreover, models that integrate care to treat people with mental health and medical comorbidities have proven effective, but despite their effectiveness, these models are not in widespread use.".2 More research is needed to identify best practices regarding integrated behavioral and mental health care in primary care, as well as identify barriers to adoption of these best practices into primary care practices and communities.

\section{Training Future Investigators}

One piece critical to the successful engagement and development of primary care research is the constraint of not having an adequate cadre of well-trained researchers. We believe there is a need to deliberately promote this training.

NAPCRG's hope is that by sharing these priorities, funding agencies will take them into consideration when determining where funding dollars will be allocated over the coming years.

NAPCRG's Researcb Advocacy Committee

\section{References}

1. Partnership for Solutions. A Project of Johns Hopkins University and the Robert Wood Johnson Foundation. Multiple chronic conditions: complications in care and treatment. http://www.partnershipforsolutions.org/DMS/files/2002/multiplecoitions.pdf.

2. Goodall S, Druss BG, Walker ER. Mental disorders and medical comorbidity. Robert Woods Johnson Policy Brief no. 21. 2011. http:// www.rwjf.org/content/dam/farm/reports/issue_briefs/2011/rwjf69438.

\section{AHRQ UPDATES ON PRIMARY CARE RESEARCH: SELF-MANAGEMENT SUPPORT RESOURCE LIBRARY AND COMPANION VIDEOS}

As the rates of chronic diseases in the United States rise and the number of people living with multiple chronic conditions increases, self-management support (SMS) is increasingly recognized as a fundamental strategy for improving health outcomes, reducing health care utilization, and improving quality of care. Self-management support is defined by the Institute of Medicine as "the systematic provision of education and supportive interventions by health care staff to increase patients' skills and confidence in managing their health problems, including regular assessments of progress and problems, goal setting, and problemsolving support."1 As part of its ongoing work to improve primary care practice and care for people living with multiple chronic conditions, the Agency for Healthcare Research and Quality (AHRQ) sponsored the development of 2 SMS resources for primary care clinicians and their teams. The first is a one-stop multimedia library of SMS educational materials and resources, and the second is a series of companion videos that demonstrate SMS skills and concepts and illustrate SMS in action. These robust resources can help health care teams in small and large practices visualize and adopt SMS techniques in practice.

\section{AHRQ's Self-Management Support Resource Library}

The SMS Resource Library contains 39 multimedia and action-oriented educational materials from 21 different sources. It consists of resource guides, articles, toolkits, videos, tip sheets, and tools. Materials are organized using the following framework to allow clinicians to easily identify which materials best suit their needs:

- What is self-management support: Includes resources that define and describe SMS

- Why is self-management support important: Includes success stories from patients, evidence on SMS (eg, peer-reviewed manuscripts), and benefits of implementing SMS

- How to implement self-management support into practice: Includes tools that providers and clinical teams can use with their patients to encourage health-related behavior change

\section{Motivational Videos on Self-Management Support}

Three brief videos illuminate SMS skills and concepts, show its use in diverse primary care practices, and feature clinicians who have successfully implemented SMS. The video vignettes demonstrate the core competencies of SMS for clinicians, as defined by the New Health Partnerships. These competencies are: Emphasize Patient Role; Build Relationships; Include Family; Share Information; Collaborate on Agenda Setting, Goals, and Action Plans; Problem Solving; and Follow Up. ${ }^{2}$

More information about implementing selfmanagement support in primary care settings is available on AHRQ's Improving Primary Care Practice web pages. The AHRQ SMS Resource Library, including access to the SMS tools and videos is available at: http://www.orau.gov/ahrq/sms_home.html. 